\title{
MOTION GROUPS AND ABSOLUTELY CONVERGENT FOURIER TRANSFORMS
}

\author{
GARTH I. GAUDRY and RITA PINI
}

(Received 5 December 1985)

Communicated by W. Moran

\begin{abstract}
According to an extension of a classical theorem of Bernstein, due to C. Herz, a function on $\mathbf{R}^{n}$ belonging to a Besov space of appropriate order has an absolutely convergent Fourier transform. We establish extensions of this result to Cartan motion groups, for Besov spaces defined with respect to both isotropic and non-isotropic differences.
\end{abstract}

1980 Mathematics subject classification (Amer. Math. Soc.): 22 E 30, 41 A 17, 43 A 50, 43 A 80.

\section{Introduction}

Let $(G, K)$ be a Riemannian symmetric pair of compact type, with corresponding involution of $G$ denoted by $\tilde{\theta}$ [2, Chapter IV]. Denote by $\theta$ the derived involution of the Lie algebra $g$, and set

$$
k=\{X \in g: \theta(X)=X\}, \quad V=\{Y \in g: \theta(Y)=-Y\} .
$$

Then the subspace $V$ is stable under the Adjoint action of $K$, and one can define the Cartan motion group $K \ltimes V$ in which the law of multiplication is given by the formula

$$
\left(k_{1}, Y_{1}\right) \cdot\left(k_{2}, Y_{2}\right)=\left(k_{1} k_{2}, Y_{1}+\operatorname{Ad}\left(k_{1}\right) Y_{2}\right)
$$

This research was partially supported by the Consiglio Nazionale delle Ricerche, G.N.A.F.A. The research of the first author was supported by the Australian Research Grants Scheme. - 1987 Australian Mathematical Society 0263-6115/87 \$A2.00+0.00 
The group $M=K \ltimes V$ is a Lie group whose Lie algebra $m$ is $k+V$ (as a vector space), in which the Lie product is given by the formulas

$$
[X, Y]_{m}=\left\{\begin{array}{l}
0 \quad \text { if } X, Y \in V \\
{[X, Y]_{g} \text { if } X \in k, Y \in m}
\end{array}\right.
$$

It is well known, and easy to verify, that the exponential map, and one-parameter subgroups, are given by the formula

$$
\exp _{M}(t X, t Y)=\left(\exp _{K}(t X), \frac{e^{t a d X}-I}{X} \cdot Y\right)
$$

In (1), $t \in R, X \in k, Y \in V$, and

$$
\frac{e^{t a d X}-I}{X} \cdot Y=\sum_{n=1}^{\infty} t^{n}(\operatorname{ad} X)^{n-1} \cdot Y .
$$

We shall suppose fixed a norm on $g$ which is Ad-invariant. With respect to such a norm, $k$, and $V$ are orthogonal.

The best known examples of Cartan motion groups are the groups $M(n)$, $(n \geqslant 2)$. These correspond to the choice $G=S O(n+1), K=S O(n)$, and $\tilde{\theta}$ the involution of $G$ corresponding to the matrix

$$
\left(\begin{array}{cc}
I_{n} & \\
& -1
\end{array}\right)
$$

The subspace $V$ of $S O(n+1)$ is the set of matrices with 0 in the upper-left $n \times n$ block; it may be identified with $\mathbb{R}^{n}$; the Adjoint action of $k \in S O(n)$ on $v \in V$ is the rotation of $v$ by amount $k$. See [2] for details.

Our aim is to establish a theorem of Bernstein type for the Cartan motion groups. To do so, it is necessary to define a notion of "difference", modulus of continuity, and Besov space of appropriate type. We shall show that a recursive definition of difference works perfectly for $M(2)$, but that the methods that appear so natural and work well for $M(2)$ cannot be made to function even for $M(3)$. On the other hand, a nonrecursive (that is, non-isotropic) definition of difference works well in general. In the latter case, the argument follows the general line of Inglis [4] (for the Heisenberg group).

The theorems we prove are sufficient to establish that functions of an appropriate degree of smoothness and decay at infinity belong to the Fourier algebra $A(G)$. 
A theorem of Bernstein type for compact, connected Lie groups (using isotropic differences) is to be found in [1]. A fundamental paper regarding (inter alia) a Bernstein theorem for $\mathbb{R}^{n}$ (and $\mathbb{T}^{n}$ ) is Herz [3].

\section{Isotropic differences}

Definition. (i) If $k$ is a positive integer, $(X, Y) \in m=k+V$, and $f$ is a measurable function on $M$, we define the $k$ th difference of $f$ determined by the direction $(X, Y)$ as

$$
\begin{aligned}
\Delta_{(X, Y)}^{k} f(x, y) & =\sum_{j=0}^{k}(-1)^{k+j} C(k, j) f((x, y) \exp (j X, j Y)) \\
& =\sum_{j=0}^{k}(-1)^{k+j} C(k, j) f\left((x, y)\left(\exp _{K} j X, \frac{e^{j \mathrm{ad} X}-I}{X} \cdot Y\right)\right) .
\end{aligned}
$$

The coefficients $C(k, j)$ are the binomial coefficients $\left(\begin{array}{l}k \\ j\end{array}\right)$.

(ii) If $1 \leqslant p \leqslant \infty$, define

$$
\omega_{k}\left(t, f, L^{p}\right)=\sup _{0<\| K X, Y) \|<t}\left\|\Delta_{(X, Y)}^{k} f\right\|_{p} .
$$

(iii) If $\alpha, \beta>0$, and $k>\max (\alpha, \beta)$, define

$$
N_{(\alpha, \beta, p)}^{k}(f)=\int_{0}^{1} \frac{\omega_{k}\left(t, f, L^{p}\right)}{t^{\alpha+1}} d t+\int_{1}^{\infty} \frac{\omega_{k}\left(t, f, L^{p}\right)}{t^{\beta+1}} d t .
$$

Let $\Lambda_{(\alpha, \beta, p)}^{k}(M)$ be the completion of $C_{c}^{\infty}(M)$ with respect to the "norm" $N_{(\alpha, \beta, p)}$.

DEFINITION. Let $A(M)$ denote the space of bounded continuous functions $f$ on $M$ which can be written as the sum of a uniformly convergent series

$$
f=\sum_{j=1}^{\infty} g_{j} * h_{j}
$$

in which $g_{j}, h_{j} \in L^{2}(M)$, and

$$
\sum_{j=1}^{\infty}\left\|g_{j}\right\|_{2}\left\|h_{j}\right\|_{2}<+\infty
$$

The norm of $f$ in $A(M)$ is the infimum of all sums (3) with respect to all possible representations (2). 


\section{The case of $M(2)$}

Let $X_{1}, X_{2}, X_{3}$ denote the standard basis elements of $m(2)$ :

$$
X_{1}=\left(\begin{array}{rrr}
0 & -1 & 0 \\
1 & 0 & 0 \\
0 & 0 & 0
\end{array}\right), \quad X_{2}=\left(\begin{array}{rrr}
0 & 0 & -1 \\
0 & 0 & 0 \\
1 & 0 & 0
\end{array}\right), \quad X_{3}=\left(\begin{array}{rrr}
0 & 0 & 0 \\
0 & 0 & -1 \\
0 & 1 & 0
\end{array}\right)
$$

We shall suppose that $\left\|X_{1}\right\|=1$. Observe that if $s \in \mathbb{R}$, the element $X=s X_{1}$ is a typical element of a Cartan subalgebra of so(3), that $V$ is the polarisation of a rot space of so(3), and that

$$
\begin{aligned}
& e^{\operatorname{ad} X}\left(X_{2}\right)=(\cos s) X_{2}+(\sin s) X_{3}, \\
& e^{\operatorname{ad} X}\left(X_{3}\right)=(-\sin s) X_{2}+(\cos s) X_{3} .
\end{aligned}
$$

Furthermore, the action of $\operatorname{ad}(X)$ on $V$ is represented by the matrix

$$
\left(\begin{array}{rr}
0 & -s \\
s & 0
\end{array}\right)
$$

It follows that the action of $\left(e^{\lambda a d X}-I\right) / X$ on $V$ is the composition of a rotation with a dilation of amount $\sin \frac{1}{2}\left\|\lambda X \mid / \frac{1}{2}\right\| X \|$.

THEOREM. Let $f \in C_{c}(M)$ and suppose $k>3 / 2$. Then

$$
\|f\|_{A} \leqslant C N_{(3,2,2)}^{k}(f)
$$

where the constant $C$ does not depend on $f$ (or on its support).

Proof. We identify $S O(2)$ with the circle group $\pi=\left\{e^{i \theta}:-\pi<\theta \leqslant \pi\right\}$. Then the Haar measure on $S O(2) \ltimes \mathbb{R}^{2}$ is the product of the respective Haar measures.

Observe that if $F_{n}$ is a function on $S O(2), \phi_{n}$ a radial (that is, $\operatorname{Ad}(K)$-invariant) function on $\mathbb{R}^{2}, \psi_{n}$ a function on $S O(2)$, and $H_{n}$ is the function on $M(2)$ given by the formula

$$
H_{n}\left(e^{i t}, v\right)=F_{n}\left(e^{i t}\right) \phi_{n}\left(\psi_{n}\left(e^{i t}\right) v\right),
$$

then (under suitable conditions)

$$
H_{n} * H_{m}=H_{m} * H_{n}
$$

for all $m, n$. 
In particular, we construct a commuting family of functions $\left\{h_{n}\right\}$ on $M(2)$ as follows

$$
h_{n}\left(e^{i t}, v\right)=\left\{\begin{array}{c}
c_{n}^{-1} \sum_{j=1}^{k}(-1)^{k+j} \frac{1}{j^{3}} C(k, j) F_{n}\left(e^{i / / j}\right) \phi_{n}\left(\frac{\frac{t}{2}}{\sin \frac{l}{2}}\left(\frac{v}{j}\right)\right) \frac{t^{2}}{\sin ^{2} \frac{t}{2}}, \quad n>0, \\
c_{n}^{-1} \sum_{j=1}^{k}(-1)^{k+j} \frac{1}{j^{3}} C(k, j) F_{0}\left(e^{i t / j}\right) \phi_{n}\left(\frac{\frac{l}{2}}{\sin \frac{l}{2}}\left(\frac{v}{j}\right)\right) \frac{t^{2}}{\sin ^{2} \frac{l}{2}}, \quad n \leqslant 0,
\end{array}\right.
$$

where $F_{n}$ denotes the characteristic function of the $\operatorname{arc}\left\{e^{i \theta}:-k^{-n} \pi<\theta<k^{-n} \pi\right\}$ and $\phi_{n}$ the characteristic function of the ball of radius $k^{-n}$ in $\mathbb{R}^{2}$. The constant $c_{n}$ is to be chosen so that $\int_{M(2)} h_{n}=1$. Then, thanks to the observation above,

$$
h_{n} * h_{m}=h_{m} * h_{n}
$$

for all $m, n$.

We proceed to study the asymptotic behaviour of the coefficients $c_{n}$. If $n>0$, the changes of variable $t \rightarrow j t, v \rightarrow(\sin (j t / 2) /(t / 2)) \cdot v$ yield

$$
c_{n}=\left\{\sum_{j=1}^{k}(-1)^{k+j} C(k, j)\right\}\left(k^{-n}\right)^{3}=C^{\prime}\left(k^{-n}\right)^{3}
$$

where $C$ and $C^{\prime}$ are constants.

Similarly, if $n \leqslant 0 ; c_{n}=C^{\prime \prime}\left(k^{-n}\right)^{2}$. Consequently, there exist constants $A, B$ $>0$ such that

$$
\begin{array}{ll}
A k^{3 n} \leqslant\left|c_{n}\right|^{-1} \leqslant B k^{3 n} & (n>0), \\
A k^{2 n} \leqslant\left|c_{n}\right|^{-1} \leqslant B k^{2 n} & (n \leqslant 0) .
\end{array}
$$

For each integer $n$, let

$$
P_{n}=h_{n}-h_{n-1} \text { and } Q_{n}=h_{n}+h_{n-1}
$$

and consider the formal series

$$
\sum_{n=-\infty}^{\infty} f * P_{n} * Q_{n}
$$

whose $N$ th symmetric partial sum is, thanks to (4), (6), $f * h_{N} * h_{N}-$ $f * h_{-N} * h_{-N}$. Since $h_{N}$ is an approximate identity as $N \rightarrow \infty$, it follows that $f * h_{N} * h_{N} \rightarrow f$ uniformly. On the other hand, $\left\|f * h_{-N} * h_{-N}\right\|_{\infty} \leqslant\|f\|_{1}\left\|h_{-N}\right\|_{2}^{2}$. It is a simple matter to check that $\left\|h_{-N}\right\|_{2} \rightarrow 0$ as $N \rightarrow+\infty$. Therefore the series (7) represents $f$ in the uniform sense.

The final stage of the proof is the estimation of $\sum_{-\infty}^{\infty}\left\|f * P_{n}\right\|_{2}\left\|Q_{n}\right\|_{2}$. We use $C_{1}, C_{2}, \ldots$ to denote a sequence of constants. 


\section{Consider}

(8)

$$
f * P_{n}=\left(f * h_{n}+(-1)^{k} f\right)-\left(f * h_{n-1}+(-1)^{k} f\right) .
$$

Now, if $n>0$

$$
\begin{aligned}
& \left(f * h_{n}\right)\left(e^{i x}, y\right)=c_{n}^{-1} \sum_{j=1}^{k}(-1)^{k+j} C(k, j) j^{-3} \\
& \quad \times \int_{\mathbf{T}} \int_{\mathbf{R}^{2}} f\left(\left(e^{i x}, y\right)\left(e^{i t}, v\right) F_{n}\left(e^{i t / j}\right) \phi_{n}\left(\frac{t / 2}{\sin (t / 2)}\right)\left(\frac{v}{j}\right)\right) \frac{t^{2}}{\sin ^{2}(t / 2)} d t d v .
\end{aligned}
$$

The changes of variables $t \rightarrow j t, v \rightarrow\left(e^{j \mathrm{ad} t}-I\right) / \mathrm{ad} t \cdot v$ yield

$$
\begin{aligned}
\left(f * h_{n}\right)\left(e^{i x}, y\right)= & c_{n}^{-1} \int_{\mathbf{T}} \int_{\mathbf{R}^{2}} \sum_{j=1}^{k}(-1)^{k+j} C(k, j) \\
& \times f\left\{\left(e^{i x}, y\right)\left(e^{i j t}, \frac{e e^{j \mathrm{ad} t X_{1}}-I}{t X_{1}} \cdot v\right)\right\} F_{n}\left(e^{i t}\right) \phi_{n}\left(v^{\prime}\right) d t d v
\end{aligned}
$$

where $v^{\prime}$ is a certain rotation of $v$. Since $\phi_{n}$ is rotation-invariant, $v^{\prime}$ may be replaced by $v$. It follows that

$$
\left(f * h_{n}+(-1)^{k} f\right)\left(e^{i x}, y\right)=c_{n}^{-1} \int_{-k^{-n}}^{k^{-n}} d t \int_{\|v\| \leqslant k^{-n}} d v \Delta_{\left(t X_{1}, v\right)}^{k} f\left(e^{i x}, y\right)
$$

whence

$$
\left\|f * P_{n}\right\|_{2} \leqslant C_{1}\left\{\omega_{k}\left(\sqrt{2} k^{-n}, f, L^{2}\right)+\omega_{k}\left(\sqrt{2} k^{-n+1}, f, L^{2}\right)\right\} .
$$

If $n \leqslant 0$, one obtains an estimate identical to (9).

Concerning the estimate for $\left\|Q_{n}\right\|_{2}$, we have, when $n>0$,

$$
\begin{aligned}
\left\|h_{n}\right\|_{2} & \leqslant C_{2}\left|c_{n}\right|^{-1} \sup _{j}\left\{\int_{\mathbf{T}} \int_{\mathbf{R}^{2}}\left|F_{n}\left(e^{i t / j}\right) \phi_{n}\left(\frac{t / 2}{\sin \frac{t}{2}}\left(\frac{v}{j}\right)\right) \frac{t^{2}}{\sin ^{2} \frac{t}{2}}\right|^{2} d t d v\right\}^{1 / 2} \\
& \leqslant C_{3}\left|c_{n}\right|^{-1}\left\{\int_{\mathbf{T}} \int_{\mathbf{R}^{2}}\left|F_{n}\left(e^{i t}\right) \phi_{n}(v)\right|^{2} d t d v\right\}^{1 / 2} \\
& \leqslant C_{4} k^{3 n}\left(k^{-n}\right)^{3 / 2}=C_{4}\left(k^{-n}\right)^{-3 / 2}
\end{aligned}
$$

whence

$$
\left\|Q_{n}\right\|_{2} \leqslant C_{5}\left(k^{n}\right)^{3 / 2} .
$$

If $n \leqslant 0$, similar estimates give

$$
\left\|Q_{n}\right\|_{2} \leqslant C_{6} k^{n}
$$


Collecting together (9), (10) and (11), we obtain the estimate

$$
\begin{aligned}
\sum_{-\infty}^{\infty}\left\|f * P_{n}\right\|_{2}\left\|Q_{n}\right\|_{2} & =\sum_{1}^{\infty}+\sum_{-\infty}^{0} \\
\leqslant & C_{7}\left\{\sum_{1}^{\infty}\left(\omega_{k}\left(\sqrt{2} k^{-n}, f, L^{2}\right)+\omega_{k}\left(\sqrt{2} k^{-n+1}, f, L^{2}\right)\right) k^{3 n / 2}\right. \\
& \left.\quad+\sum_{-\infty}^{0}\left(\omega_{k}\left(\sqrt{2} k^{-n}, f, l^{2}\right)+\omega_{k}\left(\sqrt{2} k^{-n+1}, f, L^{2}\right)\right) k^{n}\right\} \\
\leqslant & C_{8}\left\{\sum_{1}^{\infty} \omega_{k}\left(\sqrt{2} k^{-n}, f, L^{2}\right) k^{3 n / 2}+\sum_{-\infty}^{0} \omega_{k}\left(\sqrt{2} k^{-n}, f, L^{2}\right) k^{n}\right\} \\
& \leqslant C_{9}\left\{\int_{0}^{1} \frac{\omega_{k}\left(t, f, L^{2}\right)}{t^{(3 / 2)+1}} d t+\int_{1}^{\infty} \frac{\omega_{k}\left(t, f, L^{2}\right)}{t^{2}} d t\right\} \\
& =C_{9} N_{(3,2,2)}^{k}(f) .
\end{aligned}
$$

\section{The case $M(n)(n \geqslant 3)$ : isotropic dilations}

In the proof just given, there are, apart from the detailed estimates, two main ingredients.

(a) The construction of a commutative approximate identity of the form

$$
\sum_{j=1}^{k}(-1)^{k+j} C(k, j) h_{n}^{(j)} .
$$

(b) The transfer, by appropriate change of variables, of convolution of $f$ with an element (12), to an integral sum of differences

$$
\sum(-1)^{k+j} C(k, j) f((x, y) \exp (j(X, Y))
$$

with respect to a weight which must of course be independent of $j$ (see $\$ 2$ ).

One would expect to be able to extend the general approach of $\$ 3$ to cover all Cartan motion groups, in particular the Euclidean ones. However, as we shall now show, it does not seem to be possible to do so.

The first requirement (a) is easily enough met. One can construct a family $\left\{h_{m}^{(j)}\right\}_{j=1, \ldots, k}$ of integrable functions on $M(n)$ such that

(i) $h_{m}^{(j)}(\cdot, v)$ is central for every $v$;

(ii) $h_{m}^{(j)}(k, \cdot)$ is radial (that is, $\operatorname{Ad}(S O(n))$-invariant) for every $k \in S O(n)$. 
Then, with appropriate constraints on the size of the supports and the distribution of the values of the $h_{m}^{(j)}$,

$$
\left\{\sum_{j=1}^{k}(-1)^{k+j} C(k, j) h_{m}^{(j)}\right\}_{m-1}^{\infty}
$$

is a commutative approximate identity on $M(n)$.

The requirement (b) takes the form (see \$2)

(iii) $h_{m}^{(j)}\left(\exp j T,\left(e^{j \text { ad } T}-I\right) / T \cdot v\right)=h^{(1)}\left(\exp T,\left(e^{\operatorname{ad} T}-I\right) / T \cdot v\right)$ for $j=$ $2, \ldots, k, T \in t$ (a Cartan subalgebra of $k=S O(n)$ ), and $v \in V \simeq \mathbb{R}^{n}$.

We now show that the requirement (iii) is incompatible with (i) and (ii). We shall treat the case $M(3)$, although the argument is general. We shall suppress the subscript $M$ and show that

Proposition. There do not exist nontrivial integrable functions $h^{(1)}$ and $h^{(2)}$ on $M(3)$ satisfying (i), (ii) and (iii).

Proof. Let $t$ be the toral algebra generated by the element

$$
\left(\begin{array}{rrrr}
0 & -1 & 0 & 0 \\
1 & 0 & 0 & 0 \\
0 & 0 & 0 & 0
\end{array}\right)
$$

so so(3) $\subset$ so(4). The space $V=\mathbb{R}^{3}$ consists of matrices of the form

Then

$$
\left(\begin{array}{rrrr}
0 & 0 & 0 & -v_{1} \\
0 & 0 & 0 & -v_{2} \\
0 & 0 & 0 & -v_{3} \\
v_{1} & v_{2} & v_{3} & 0
\end{array}\right) \sim\left(v_{1}, v_{2}, v_{3}\right)=v .
$$

$$
\frac{e^{\mathrm{ad} T}-I}{T} \cdot v=2 \sin \frac{T}{2}\left(w_{1}, w_{2}, 0\right)+\left(0,0, v_{3}\right)
$$

where $\left(w_{1}, w_{2}, 0\right)$ is a rotate of $\left(v_{1}, v_{2}, 0\right)$ in the hyperplane $v_{3}=0$.

If $h^{(1)}$ and $h^{(2)}$ satisfy (iii), then

$$
h^{(2)}\left(\exp X, v^{\prime}\right)=h^{(1)}\left(\exp \frac{X}{2}, \frac{e^{\operatorname{ad}(X / 2)}-I}{X / 2} \cdot \frac{X / 2}{e^{\operatorname{ad} X}-I} \cdot v^{\prime}\right)
$$

for all $x \in t, v^{\prime} \in V$. Because of (ii),

$$
h^{(2)}\left(\exp X, v^{\prime}\right)=K
$$

say, on the sphere $\left\|v^{\prime}\right\|=C, X$ being fixed. As $v^{\prime}$ varies over the sphere of radius $C$, the vector

$$
\frac{e^{\operatorname{ad}(X / 2)}-I}{X / 2} \cdot \frac{X / 2}{e^{\operatorname{ad} X}-I} \cdot v^{\prime}
$$


varies over the ellipsoid whose equation is

$$
f(x, y, z)=\left(4 \cos ^{2} \frac{X}{4}\right) x^{2}+\left(4 \cos ^{2} \frac{X}{4}\right) y^{2}+z^{2}=C^{2} .
$$

This follows from (13). The semiaxes of the ellipsoid are $(C / 2) \cos (X / 4)$, $C / 2 \cos (X / 4), C$. For fixed $X$, the function $v \rightarrow h^{(1)}(\exp (x / 2), v)$ is rotation invariant. Therefore

$$
h^{(1)}\left(\exp \frac{x}{2}, v\right)=K
$$

as $v$ varies over the spherical shell of radii $C,(C / 2) \cos (x / 2)$. It follows from (13) and (14) that

$$
h^{(2)}(\exp X,(0,0, \gamma))=h^{(1)}\left(\exp \frac{x}{2},(0,0, \gamma)\right)=K
$$

for all $\gamma$ between $C$ and $(C / 2) \cos (X / 4)$. It therefore follows that $h^{(2)}\left(\exp (X / 2), v^{\prime}\right)=K$ for all $v^{\prime}$ in the spherical shell of radii $C,(C / 2) \cos (X / 4)$. Clearly then, $h^{(2)}\left(\exp X, v^{\prime}\right)=K$ for all $v^{\prime}$, except in the special case where $\cos (X / 4)=\frac{1}{2}$.

Since the integral of $h^{(2)}(\exp X, \cdot)$ is infinite for almost all $X$, and since $h^{(2)}$ is central with respect to the first variable, it follows that the integral of $h^{(2)}$ over $M(3)$ must be infinite.

\section{Cartan motion groups: nonisotropic differences}

Let $M=K \ltimes V$, and for simplicity, suppose that $K$ is connected. Fix a maximal torus $T$ of $K$, with corresponding Lie algebra $t$; let $P_{+}$be a choice of positive roots for $\left(t^{\mathrm{C}}, k^{\mathrm{C}}\right)$, and write $\lambda=\left|P_{+}\right|$. Denote the dimension of $M, K$ and $V$ by $\mu, k, \nu$ respectively and the rank of $K$ by $\rho$.

Then

$$
\mu=\nu+k=\nu+2 \lambda+\rho .
$$

Definition. Let $k$ be a positive integer, and $f$ a measurable function on $M$. For $(X, Y) \in k+V$, define the $k$ th order nonisotropic difference of $f, \bar{\Delta}_{(X, Y)}^{k} f$, to be the function

$$
\bar{\Delta}_{(X, Y)}^{k} f(x, y)=\sum_{j=1}^{k}(-1)^{k+j} C(k, j) f((x, y)(\exp j X, j Y)) .
$$

Observe that

$$
\bar{\Delta}_{(X, Y)}^{k} f \neq \bar{\Delta}_{(X, Y)}^{(k-1)}\left(\bar{\Delta}_{(X, Y)} f\right)
$$


Definition. For each positive integer $k, t>0$, and $1 \leqslant p \leqslant+\infty$, define

$$
\bar{\omega}_{k}\left(t, f, L^{p}\right)=\sup _{0<\| X, Y) \|<t}\left\|\bar{\Delta}_{\left(X, Y^{\prime}\right)}^{k} f\right\|_{p} .
$$

Set

$$
\bar{N}_{(\alpha, \beta, p)}^{k}(f)=\int_{0}^{1} \frac{\bar{\omega}_{k}\left(t, f, L^{p}\right)}{t^{\alpha+1}} d t+\int_{1}^{\infty} \frac{\bar{\omega}_{k}\left(t, f, L^{p}\right)}{t^{\beta+1}} d t
$$

THEOREM. Let $f \in C_{c}(M)$. Then if $k>\mu / 2$,

$$
\|f\|_{A} \leqslant C \bar{N}_{(\mu / 2, v / 2,2)}^{k}(f)
$$

where $C$ is independent of $f$.

Proof. Let $B_{0}$ be a ball centered at the origin in $t$ chosen so that the exponential mapping is regular on $B_{0}$. For each integer $n>0$, write

$$
F_{n}(T)=\chi_{B_{0}}\left(k^{n} T\right) \text {. }
$$

For each integer $n$, let $\phi_{n}$ be the characteristic function of the ball of radius $k^{-n}$ in $V$. then $\phi_{n}$ is $\operatorname{Ad}(K)$-invariant.

Define the sequence $\left\{h_{n}\right\}_{-\infty}^{\infty}$ of functions on $t+V$ as follows

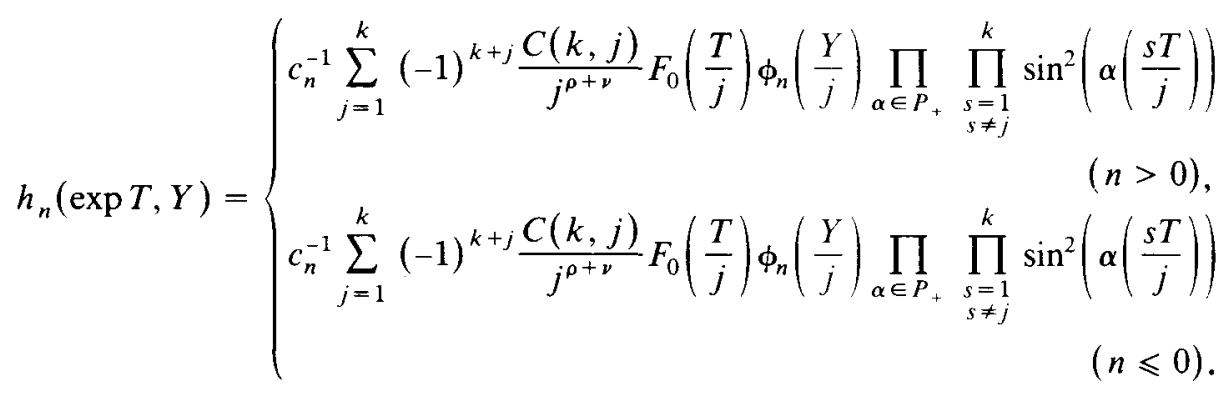

Let $\tilde{h}_{n}$ be the function on $M=K \ltimes V$ such that $\tilde{h}_{n}(\cdot, Y)$ is central on $K$ for each $Y$, and such that

$$
\tilde{h}_{n}(\exp T, Y)= \begin{cases}h_{n}(T, Y), & T \in B_{0}, \\ 0, & \text { otherwise }\end{cases}
$$

The coefficient $c_{n}$ is chosen so that

$$
\int_{M} \tilde{h}_{n} d m=1
$$


When $n>0$, the Weyl integration formula shows that

$$
\begin{aligned}
c_{n}=\sum_{j=1}^{k}(-1)^{k+j} \frac{C(k, j)}{j^{\rho+\nu}|W|} \int_{K / T} \int_{t} \int_{V} F_{n}\left(\frac{T}{j}\right) \phi_{n}\left(\frac{Y}{j}\right) & \prod_{\substack{s=1 \\
s \neq j}}^{k} \prod_{\alpha \in P_{+}} \sin ^{2}\left(\alpha\left(\frac{s T}{j}\right)\right) \\
& \times \prod_{\alpha \in P_{+}} \sin ^{2}(\alpha(T)) d Y d T d z
\end{aligned}
$$

$|W|$ being the order of the Weyl group. The changes of variables $T \rightarrow j T, Y \rightarrow j Y$ lead to the expression

$$
c_{n}=\sum_{j=1}^{k}(-1)^{k+j} C(k, j) \int_{t} \chi_{B_{0}}\left(k^{n} T\right) \int_{\|Y\| \leqslant k^{-n}} \prod_{s=1}^{k} \prod_{\alpha \in P_{+}} \sin ^{2}(\alpha(s T)) d T d V .
$$

Using the fact that $1 \geqslant(\sin \theta) / \theta \geqslant 2 / \pi$ if $0<\theta<\pi / 2$, we see that

$$
\left|c_{n}\right| \sim\left(k^{-n}\right)^{\rho+\nu+2 \lambda k} \text { as } n \rightarrow+\infty \text {. }
$$

Similarly one obtains that

$$
\left|c_{n}\right| \sim\left(k^{-n}\right)^{\nu} \text { as } n \rightarrow-\infty .
$$

Define the sequences $P_{n}$ and $Q_{n}$ by $P_{n}=\tilde{h}_{n}-\tilde{h}_{n-1}, Q_{n}=\tilde{h}_{n}+\tilde{h}_{n+1}$ and the formal series $\sum_{-\infty}^{\infty} f * P_{n} * Q_{n}$. In virtue of the central behaviour of $\tilde{h}_{n}$ with respect to the first variable, and its $\operatorname{Ad}(K)$-invariance with respect to the second, it is an easy matter to check that $\tilde{h}_{n} * \tilde{h}_{m}=\tilde{h}_{m} * \tilde{h}_{n}$ for all $m, n$. Therefore the $N$ th partial sum of the formal series is

$$
f * \tilde{h}_{N} * \tilde{h}_{N}-f * \tilde{h}_{-N} * \tilde{h}_{-N} .
$$

Much as in the case considered in section 2, this converges uniformly to $f$ as $N \rightarrow \infty$.

In order to estimate $\left\|f * P_{n}\right\|_{2}$, we calculate, for $n>0$,

$$
\begin{aligned}
f * \tilde{h}_{n}(x, y) & =\frac{c_{n}^{-1}}{|W|} \sum_{j=1}^{k}(-1)^{k+j} \frac{C(k, j)}{j^{\rho+\nu}} \int_{K / T} \int_{t} \int_{V} f\left((x, y)\left(z \exp T z^{-1}, Y\right)\right) \\
& \times F_{n}\left(\frac{T}{j}\right) \phi_{n}\left(\frac{Y}{j}\right) \prod_{\alpha \in P_{+}} \prod_{\substack{s=1 \\
s \neq j}}^{k} \sin ^{2}\left(\alpha\left(\frac{s T}{j}\right)\right) \prod_{\alpha \in P_{+}} \sin ^{2}(\alpha(T)) d z d T d Y
\end{aligned}
$$

by the Weyl integration formula. Writing $z \exp T z^{-1}=\exp (\operatorname{Ad}(z) T)$ and changing variables $T \rightarrow j T, Y \rightarrow j Y$, we obtain

$$
\begin{aligned}
f * \tilde{h}_{n}(x, y)= & \frac{c_{n}^{-1}}{|W|} \int_{K / T} \int_{t} \int_{V} \sum_{j=1}^{k}(-1)^{k+j} C(k, j) f((x, y)(\exp (j \operatorname{Ad} z T), j Y)) \\
& \times F_{n}(T) \phi_{n}(Y) \prod_{\alpha \in P_{+}} \prod_{s=1}^{k} \sin ^{2}(\alpha(s T)) d T d Y d z
\end{aligned}
$$


So

$$
\begin{aligned}
\left(f * \tilde{h}_{n}+(-1)^{k} f\right)(x, y)= & \frac{c_{n}^{-1}}{|W|} \int_{K / T} \int_{t} \int_{V} \bar{\Delta}_{(\operatorname{Ad} z T, Y)}^{k} f(x, y) \\
& \times \prod_{\alpha \in P_{+}} \prod_{s=1}^{k} \sin ^{2}(\alpha(s T)) d T d Y d z
\end{aligned}
$$

It follows that

$$
\left\|f * \tilde{h}_{n}+(-1)^{k} f\right\|_{2} \leqslant C_{1} \bar{\omega}_{k}\left(\sqrt{2} k^{-n}, f, L^{2}\right)
$$

$C_{1}$ being independent of $n$ and $f$. A similar estimate applies to the cases $n \leqslant 0$.

In estimating $\left\|Q_{n}\right\|_{2}$, for $n>0$, we note that

$$
\begin{aligned}
\left\|Q_{n}\right\|_{2} \leqslant C_{2}\left|c_{n}\right|^{-1} \sup _{1 \leqslant j \leqslant k}\left(\int_{t} d T \chi_{B_{0}}\left(k^{n} T\right) \int_{\|Y\| \leqslant k^{-n}} \prod_{\substack{s=1 \\
s \neq j}}^{k} \prod_{\alpha \in P_{+}}\right. \\
\left.\times \sin ^{2}\left(\alpha\left(\frac{s T}{j}\right)\right) \prod_{\alpha \in P_{+}} \sin ^{2}(\alpha(T))\right)^{1 / 2} .
\end{aligned}
$$

Consequently, using (16)

$$
\begin{aligned}
\left\|Q_{n}\right\|_{2} & \leqslant C_{3}\left(k^{-n}\right)^{2 \lambda k-\lambda+\rho / 2+\nu / 2}\left|c_{n}\right|^{-1} \\
& \leqslant C_{4}\left(k^{-n}\right)^{-1 / 2} .
\end{aligned}
$$

If $n \leqslant 0$, we obtain, using (17),

$$
\left\|Q_{n}\right\|_{2} \leqslant C_{5}\left(k^{-n}\right)^{-1 / 2}
$$

Finally then

$$
\begin{aligned}
\sum_{-\infty}^{\infty}\left\|f * P_{n}\right\|_{2}\left\|Q_{n}\right\|_{2}=\sum_{-\infty}^{0} \sum_{1}^{\infty} \\
\leqslant C_{6}\left(\sum_{1}^{\infty} \bar{\omega}_{k}\left(\sqrt{2} k^{-n}, f, L^{2}\right)\left(k^{-n}\right)^{-1 / 2 \mu}+\sum_{-\infty}^{\infty} \bar{\omega}_{k}\left(\sqrt{2} k^{-n}, f, L^{2}\right)\left(k^{-n}\right)^{-1 / 2 \nu}\right) \\
\leqslant C_{7}\left(\int_{0}^{1} \frac{\bar{\omega}_{k}\left(t, f, L^{2}\right)}{t^{\mu / 2+1}} d t+\int_{1}^{\infty} \frac{\bar{\omega}_{k}\left(t, f, L^{2}\right)}{t^{\nu / 2+1}} d t\right)=C_{7} \bar{N}_{(\mu / 2, v / 2,3)}^{k}(f)
\end{aligned}
$$

Concluding Remarks. We have not enunciated the theorems of Sections 2 and 5 with the degree of generality that the methods of proof would cover. For instance $f$ need not be continuous: it would suffice if $f$ were bounded and measurable with compact support. The compactness of the support is not essential either, as that part of the hypotheses is used only to conclude that $f * h_{n} \rightarrow 0$ 
as $n \rightarrow-\infty$. It would be enough for $f$ to have an appropriate kind of decay at infinity and then we would have that $f * h_{n} \rightarrow 0$, at least locally uniformly, as $n \rightarrow-\infty$.

The reader may find it useful to compare these observations with the presentation in [3].

\section{References}

[1] G. I. Gaudry and R. Pini, 'Bernstein's theorem for compact, connected Lie groups', Math. Proc. Cambridge. Philos. Soc. 99 (1986), 297-305.

[2] S. Helgason, Differential geometry and symmetric spaces, (Academic Press, New York and London, 1962).

[3] C. S. Herz, 'Lipschitz spaces and Bernstein's theorem on absolutely convergent Fourier transforms', J. Math. Mech. 18 (1968), 283-323.

[4] I. R. Inglis, 'Bernstein's theorem and the Fourier algebra of the Heisenberg group', Boll. Un. Math. Ital. A (6) 2 (1983), 39-46.

School of Mathematical Sciences

Via Lattanzio 16

The Flinders University of South Australia

20127 Milano

Bedford Park S.A. 5042

Italy

Australia 